\title{
The Physics of Mind-Matter Interaction at a Distance
}

\begin{abstract}
Luciano Pederzoli ${ }^{1,2}$, William Giroldini ${ }^{1,2}$, Elena Prati ${ }^{1}$ and Patrizio Tressoldi ${ }^{2}$
\section{ABSTRACT}

The aim of this work is identification and localisation of the interaction between mind and matter, specifically with respect to random number generators, and identification of the type of energy that can alter the degree of randomness of bit-string outputs of these electronic devices. Regarding localisation of the mind/random-numbergenerator interaction, we believe it occurs through the production of electron+gap pairs in the inversely polarised $\mathrm{P}-\mathrm{N}$ junction of the Zener diode that is used as a white noise generator, with resulting peaks of non-random current. Conversely, regarding the type of energy acting on the analogue signal, we believe it is made of photons of wavelength ranging from 0.2 to $1.1 \mu \mathrm{m}$, each therefore carrying an energy of between 6.2 and $1.14 \mathrm{eV}$. The most controversial part concerns the means by which the human mind can produce this type of energy from a distance to act directly on a chosen target, in that it is not possible for it to have been emitted by either the body or brain as biophotons.
\end{abstract}

Key Words: Mind-Matter Interaction; Biophotons; Photons-Electrons Interaction; Entanglement DOI Number: 10.14704/nq.2017.15.3.1063 NeuroQuantology 2017; 15, 3:114-119

\section{Introduction}

The aim of this current work is to outline the physical mechanisms that allow the human mind to influence from a distance, the functioning of electronic devices, specifically generators of fairly long sequences of random 0 and 1 states, known as Random Number Generators (RNGs).

Although evidence supporting the theory that the human mind is able to influence from a distance (in the absence of direct contact) random events generated by electronic devices is not yet sufficient for this phenomenon to be considered real, we believe it appropriate to ponder possible physical means through which it may occur.

Results from studies relating to the willful influence of RNGs by experienced subjects show, even with a large variability due to different experimental protocols and individual differences of these subjects, that there is indeed evidence of a change in the expected degree of randomness (see meta-analysis of Bösch, Steinkamp, \& Boller, 2006, although criticized by Kugel, 2011, as well as Radin, Nelson, Dobyns, \& Houtkooper, 2006; see also Varvoglis \& Bancel, 2016 and Tressoldi et al., 2014).

Regardless of the type of RNG used, any disruption in the generation of random 0 and 1 sequences must be a result of action upon physical events at the point where these sequences originate, and therefore must involve a physical event, albeit one produced by a mental process.

In the following paragraphs, we will try to make clear even to those not well-versed in electronics where this influence to the RNG's function could occur and what type of influence would be required.

\footnotetext{
Corresponding author: Patrizio Tressoldi

Address: ${ }^{1}$ EvanLab, Firenze, Italy; ${ }^{2}$ Science of Consciousness Research Group, Dipartimento di Psicologia Generale, Università di Padova, Italy

e-mail $\bowtie$ patrizio.tressoldi@unipd.it

Relevant conflicts of interest/financial disclosures: The authors declare that the research was conducted in the absence of any commercial or financial relationships that could be construed as a potential conflict of interest.

Received: 23 June 2017; Accepted: 26 July 2017
} 
Sequence of physical events for the generation of random series of $\mathbf{0}$ and $\mathbf{1}$ states in RNGs.

Low voltage Zener diodes, which are based on a P$\mathrm{N}$ junction in a monocrystalline ${ }^{1}$ semiconductor, produce a type of signal called "white noise".

When a continuous current is applied, the Zener diode's ends have a constant voltage of several volts (called Zener Voltage, usually from 3 to $5 \mathrm{~V}$ ) that remains virtually constant when the current across the diode is varied. Because of this feature, it is usually used as a voltage stabilizer, but at its ends another alternating voltage overlapping the constant one is created (much smaller than the Zener Voltage and usually ignored), the waveform of which is shown in Figure 2, or its expanded version in Figure 3A, in which the solid horizontal line represents its average value. The sum of the areas above the average value line is the same as the sum of areas below it.

White noise is completely random and so its voltage over time is unpredictable; all we can know about it, is that the average value is zero and effective value is non-zero - that is, able to exert power on a resistive charge.

To obtain sequences of 0 and 1 states, white noise is amplified, filtered, and introduced into a voltage comparator which (Fig 3B) emits a particular state, in this case 1 = black when the signal surpasses its average and the opposite, $0=$ white, when the signal falls below average. These variable length 0 and 1 states are then converted to an orderly string of bits by a sampler (see Fig 3C), which instantly reads and records the comparator's output at regular intervals, for example once every 500 microseconds. Since there are one million microseconds in a second, this effectively means 2000 samples per second, converted to 10 strings of 200 bits each per second.

\section{Where does the external influence have to occur to alter the RNG's random sequence?}

Theoretically, in order to alter a random sequence of 0 and 1 states, an external influence could intervene in three distinct phases of the RNG's function:

1. Directly on the waveform at the diode ends, randomly increasing or decreasing the current flowing in it.

2. On the linear electronics (specifically on the first amplifier stage), changing its response to the analogue signal applied to it.
3. On the digital electronics, changing the trigger thresholds and thus generating logic states that do not obey statistical norms.

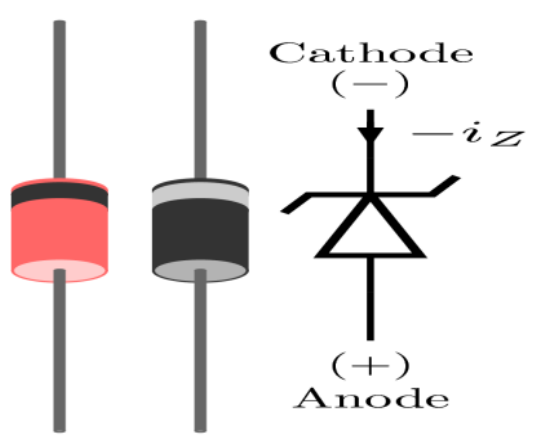

Figure 1. Example of a Zener diode and its symbol.

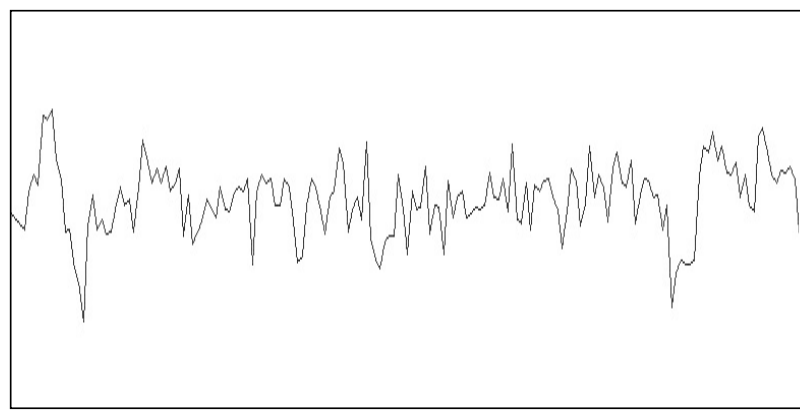

Figure 2. Example of white noise produced by a Zener diode. Time is on the horizontal ( $x$ ) axis, voltage represented on the y-axis.

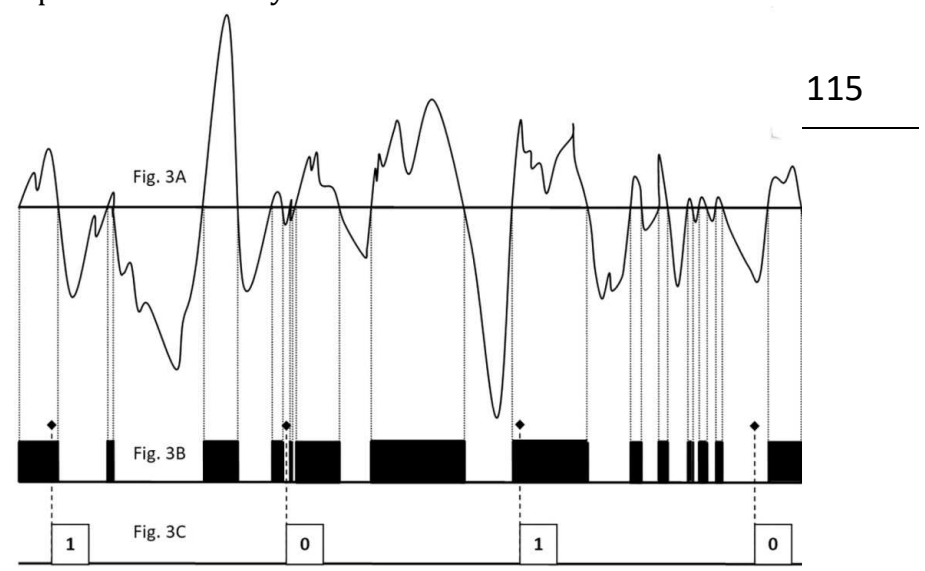

Figure 3. 3A - white noise (time on $\mathrm{x}$-axis, voltage $\mathrm{y}$-axis) with its average value (solid horizontal line); $3 \mathrm{~B}$ - voltage comparator output, showing when the analogue signal goes above or below its average; $3 \mathrm{C}$ - sequence of 0 and 1 states from the voltage sampler output, which samples at regular intervals.

Considering that, to date, the observed effects of the mind-matter interaction are rather weak, option 3 is the least probable, given that digital electronics have a lower sensitivity to noise than linear electronics.

With respect to option 2 , from the study by Tressoldi, Pederzoli, Matteoli, Prati, \& Kruth,

\footnotetext{
${ }^{1}$ https://en.wikipedia.org/wiki/P\%E2\%80\%93n_junction
} 
(2016) that illustrated the possibility of generating (from a distance) photons of wavelength 200 to $400 \mathrm{~nm}$ (i.e., from 0.2 to 0.4 $\mu \mathrm{m})$, it was apparent that the linear electronics responsible for amplifying current impulses generated by the photomultiplier tube corresponding to each counted photon did not experience significant external influences when the shutter was closed. This fact leads us to speculate that the mind "created" photons in front of the photomultiplier tube's window, but from a certain distance, such that the shutter in front of the window would let them through (if open) or block them (if closed). It therefore becomes highly likely that the influence may occurs directly on electrons that need to overcome the potential barrier of the diode's P-N junction for the quantum tunnel effect ${ }^{2}$, activating the avalanche effect ${ }^{3}$ (Figure 4).

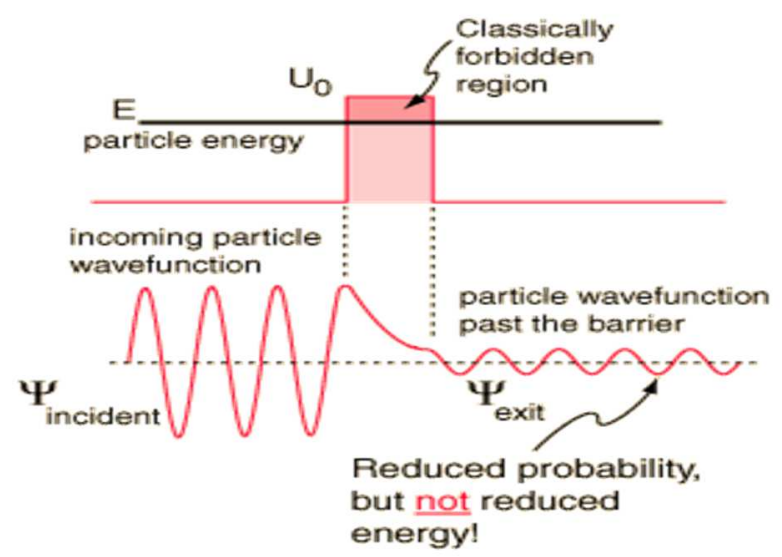

Figure 4. schematic diagram of the tunnel effect 4

\section{What type of energy does the human mind need to produce to influence RNG function?}

Let us therefore examine option 1 . If the mind influences the probability of some electrons overcoming the potential barrier of the diode's P$\mathrm{N}$ junction, then what type of energy should it produce?

With reference to observations from the aforementioned study by Tressoldi, Pederzoli, Matteoli, Prati, \& Kruth, (2016), we can theorize that the human mind is able to produce photon bursts in the range 200 to $400 \mathrm{~nm}$, with each photon's energy ranging from 3.1 to $6.2 \mathrm{eV}$ (see Appendix - Note 2). This is much greater than that needed to break one of the four covalent bonds binding each silicon atom to its neighbours in the lattice of the doped P-N monocrystal forming the
Zener diode's junction (the energy gap for silicon is $1.14 \mathrm{eV}$ ), allowing the generated electric charge to overcome the diode junction's potential barrier and produce a small current peak.

How can energy produced by the mind modify a random sequence of 0 and 1 states?

Let us now look at how a random 0 and 1 sequence could be altered, starting from modifying the flux of electrons that overcome the diode P-N junction's potential barrier. Figures 5 and 6 show how the analogue signal's average value (blue or red dotted line) can shift higher or lower as a result of an increase or decrease induced by mental energy - of the signal's amplitude (solid blue or red line), in the form of an impulse with a not insignificant area.

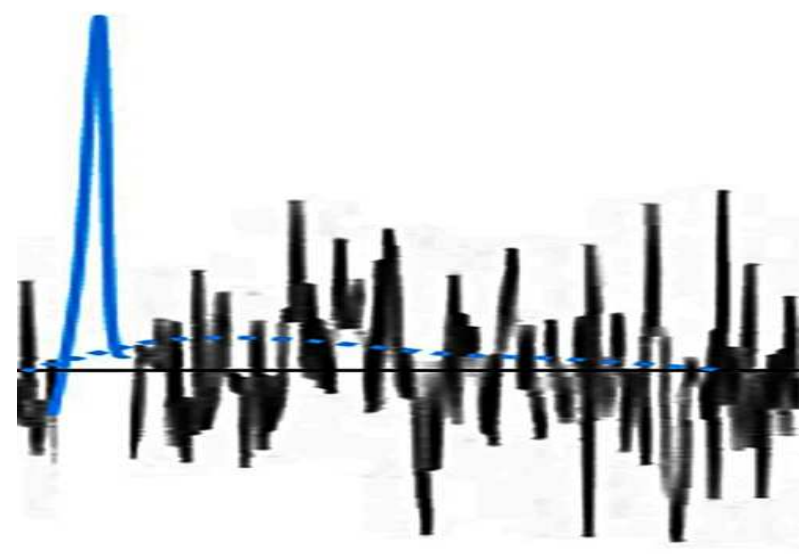

Figure 5.

116

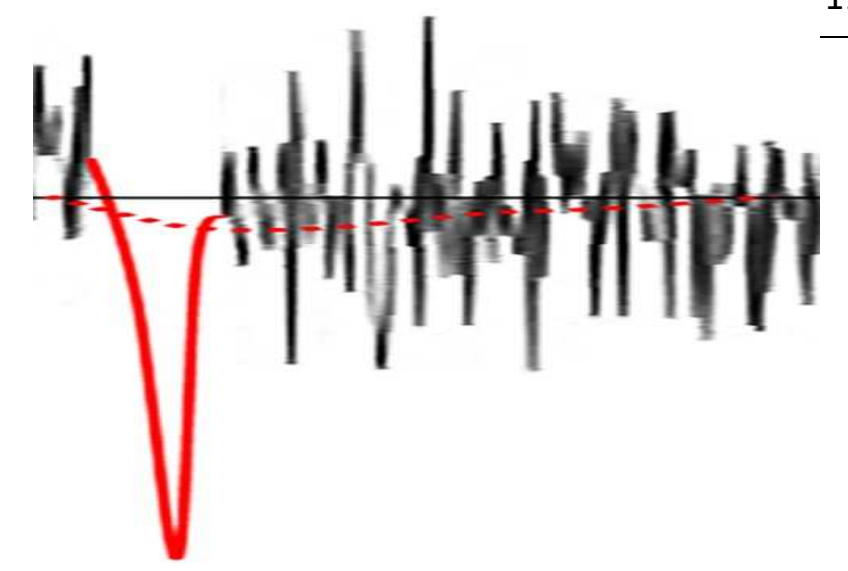

Figure 6.

If the average moves upward (Figure 7 , blue dashed line) relative to its usual value (solid black line), the duration of the detected 1 state decreases, whereas it increases if the average moves down (Figure 7, dashed red line).

The output samples that end up composing a regular sequence of random bits (1 or 0 ) can therefore produce an increase in the

3 https://www.britannica.com/science/avalanche-effect

4 https://physics.stackexchange.com/questions/93324/fromwhere-does-a-particle-get-the-energy-to-tunnel 
probability of sampling a 0 state rather than a 1 state if the average moves up, or sampling a 1 rather than a 0 state if the average goes down.

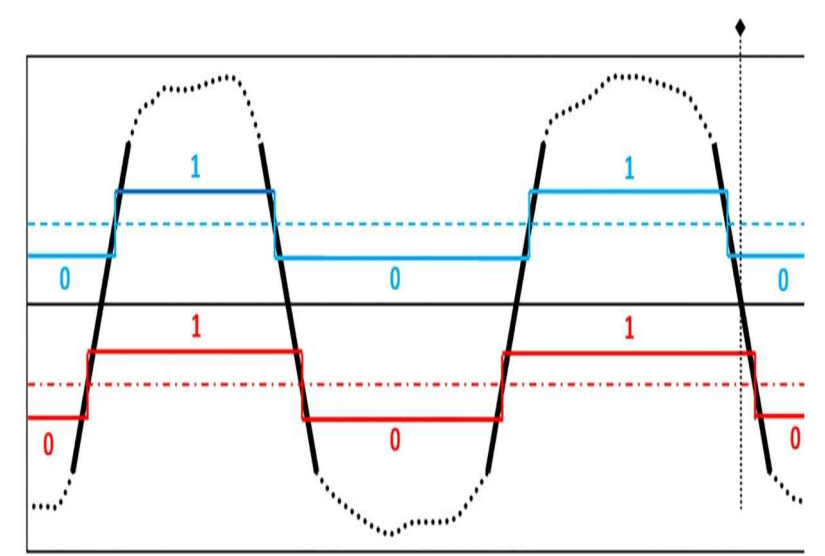

Figure 7. Effects of reduction in randomness of 1 and 0 states when analogue signal's average is increased or decreased (time on $\mathrm{x}$-axis, voltage on y-axis).

An increase in the number of charges that cross the diode's $\mathrm{P}-\mathrm{N}$ junction in interdiction state nonetheless entails an increase in current and a resulting reduction in voltage at the diode's ends; it seems more likely therefore that mental influence manifests itself in the form of negative voltage peaks.

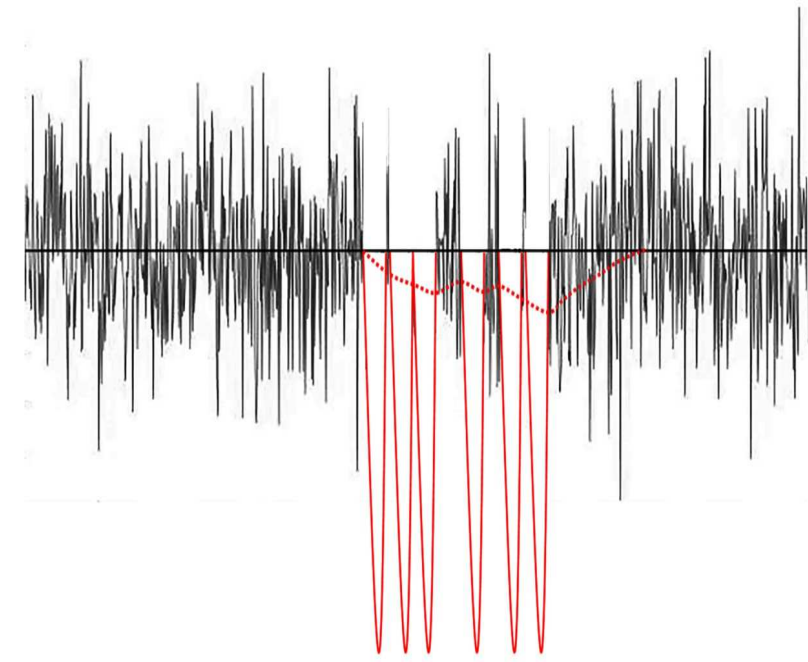

Figure 8. Example of a repeated disruption of the analogue signal - following mental intention - such that it produced a REG output with predominantly 0 states (relative to 1 states) due to the relatively long duration and low amplitude of negative peaks (time on $\mathrm{x}$-axis, voltage on $\mathrm{y}$ axis).

Obviously, the greater the energy produced by the mind, the greater the reduction in randomness of the recorded 0 and 1 sequences during sampling. In the study by Tressoldi, Pederzoli, Matteoli, Prati, \& Kruth, (2016) it was in fact observed that the energy produced by the human mind expresses itself in bursts of at least 20 photons per second within a band from 0.2 to
$0.4 \mu \mathrm{m}$. However, it is fair to theorize that it can produce bursts with higher numbers of less energetic photons, still always with an energy above $1.14 \mathrm{eV}$ and hence a wavelength less than $1.1 \mu \mathrm{m}$ (see Example 3 in Appendix - Note 2).

Nevertheless, in order to explain how with only negative peaks - it is possible to equally induce either a statistical excess of 1 states or an excess of 0 states, we must note that (see Fig. 8, with six negative peaks close together, produced by current increases from mental influence) during and after the peaks, the average value undergoes significant variation (Fig. 8 red dotted line) compared to the normal black solid line representing the average value of white noise.

When the analogue signal is above the dotted red line, the REG's voltage comparator detects a 1 state; when the signal is below the red dotted line, a 0 state is detected. During the time that the peaks are negative the comparator detects a 0 state, but in the absence of negative peaks the average is pushed down and so, as we have seen, more 1 states are detected, thus distorting their normally equal probabilities. Consequently, tall narrow peaks can statistically alter the bit sequence output in favor of 1 states, while peaks of equal area (i.e., equal energy), but lower and wider, statistically alter bit output in favor of 0 states.

\section{Discussion}

If the human mind is indeed capable of modifying the degree of randomness of RNG signals, it is necessary to identify both the nature of this interference and where it occurs. In this paper, we suggest that this interference may produce electron+gap pairs (see Appendix - Note 1) at the inversely polarized $\mathrm{P}-\mathrm{N}$ junction of the Zener diode which is used as a white noise generator, with resulting non-random current peaks.

Furthermore, we theorize that the energy produced by the human mind materializes in the form of UV photons of wavelength in the band from 0.2 to $1.1 \mu \mathrm{m}$, and thus is able to produce electron+gap pairs which generate a current in a Zener diode's P-N junction like those used in REG/RNGs (even though, due to the properties of the photomultiplier used, we have so far only measured the highest energy photons $(0.2-$ $0.4 \mu \mathrm{m})$.

Depending on whether there is an increase or decrease in the analogue signal's average, samples record either an excess or reduction in 0 states compared to 1 states, therefore reducing the randomness. This leads us to believe that 
being able to specifically influence either the increase or decrease of one of these states is more complex than simply altering their random sequence, regardless of whether the increase is of 0 or 1 . What is suggested here may also be simulated by substituting the photon bursts presumed to be created by the mind with artificially-produced photon bursts, such as from a laser.

We understand that the most critical point in our proposal is the idea of the human mind being able to generate photons from a distance and directing them to a desired target through simple intention on the part of either an individual or group of people.

While solid evidence that the body and mental activity generate biophotons is continually being gathered (Van Wijk, Van Wijk, van Wietmarschen, \& Greef, 2014; Bókkon, Salari, Tuszynski, \& Antal, 2010; Jung et al., 2005; Sun et al., 2009), it is necessary to postulate quantumlike mind-matter entanglement mechanisms to explain how it is possible for the human mind to generate photons in a target object even many kilometers away. It is in fact implausible for emanated biophotons to overcome all physical barriers between the source and the target.

A type of mind-matter entanglement becomes necessary to explain the long-distant action of the mind on a target (RNG or photomultiplier). This type of energy may effectively induce a change in the energy of the vacuum around the target, creating virtual particle-antiparticle pairs that can decay into real photon pairs (the photon is its own antiparticle). This process however implies that the mind (or more accurately: consciousness) can act as a Quantum Field, with non-local properties, in a similar way to the quantum fields (electromagnetic, gravitational, Higgs field, etc.) that current physics presumes to be the foundation of physical reality.

Walach, Tressoldi, \& Pederzoli, (2016) attempt a theoretical overview and include phenomena like this and others within the field of the Generalized Quantum Theory, which aims to generalize foundational theoretical principles of quantum mechanics not only to physical, but also biological and mental phenomena. Obviously, there is still much theoretical and empirical work to do, but we believe that to this end, our proposal is a small step forward.

\section{References}

Bókkon I, Salari V, Tuszynski JA, Antal I. Estimation of the number of biophotons involved in the visual perception of a single-object image: Biophoton intensity can be considerably higher inside cells than outside. Journal of Photochemistry and Photobiology B: Biology 2010;100(3):160-6.

Bösch H, Steinkamp F, Boller E. Examining psychokinesis: The interaction of human intention with random number generators:A meta-analysis. Psychological Bulletin 2006;132(4):497-523.

Jung H-H, Yang J-M, Woo W-M, Choi C, Yang J-S, Soh KS. Year-long biophoton measurements: normalized frequency count analysis and seasonal dependency. Journal of Photochemistry and Photobiology B: Biology 2005;78(2):149-54

Kugel W. A Faulty PK Meta-Analysis. Journal of Scientific Exploration 2011;25(1):47-62.

Radin D, Nelson R, Dobyns Y, Houtkooper J. Reexamining psychokinesis: Comment on Bösch, Steinkamp, and Boller (2006). Psychology Bulletin 2006;132(4):529-32.

Sun Y, Wang C, Dai J. Biophotons as neural communication signals demonstrated by in situ biophoton autography. Photochemical \& Photobiological Sciences 2010; 9: 315-22.

Tressoldi P, Pederzoli L, Matteoli M, Prati E, Kruth JG. Can Our Minds Emit Light at $7300 \mathrm{~km}$ Distance? A Pre-Registered Confirmatory Experiment of Mental Entanglement with a Photomultiplier. NeuroQuantology 2016;14(3):447-55.

Tressoldi PE, Pederzoli L, Caini P, Ferrini A, Melloni S, Richeldi D, Richeldi F, Duma GM. Mind-Matter Interaction at a Distance of $190 \mathrm{~km}$ : Effects on a Random Event Generator Using a Cutoff Method. NeuroQuantology 2014;12(3):337-43.

Van Wijk R, Van Wijk EPA, van Wietmarschen HA, Greef $\mathrm{J}$ van der. Towards whole-body ultra-weak photon counting and imaging with a focus on human beings: A review. Journal of Photochemistry and Photobiology B: Biology 2014;139:39-46

Varvoglis M, Bancel PA. Micro-Psychokinesis: Exceptional or Universal? Journal of Parapsychology 2016;80(1):37-44.

Walach H, Tressoldi P, Pederzoli L. Mental, behavioural and physiological nonlocal correlations within the Generalized Quantum Theory framework. Axiomathes 2016;26(3):313-28. 


\section{Appendix}

\section{Note 1}

Silicon is the most used semiconductor in electronics, including Zener diodes. It is used in monocrystalline form, comprising of a lattice in which each silicon atom - it has a valency of 4 electrons - is bound to four others by covalent bonds. If one of these bonds is broken, for example by a photon carrying an energy equal to or greater than the "energygap" (for silicon it is $1.14 \mathrm{eV}$ ), and electron+gap pair is created. In actual fact, the electron involved is released and leaves a space (absence of an $\mathrm{e}^{-}$), which is then immediately filled by an electron from an adjacent atom. The space left by this electron is in turn quickly filled by another electron from an adjacent atom, and so on, giving rise to a current in the presence of an electric field.

\section{Note 2}

Given that:

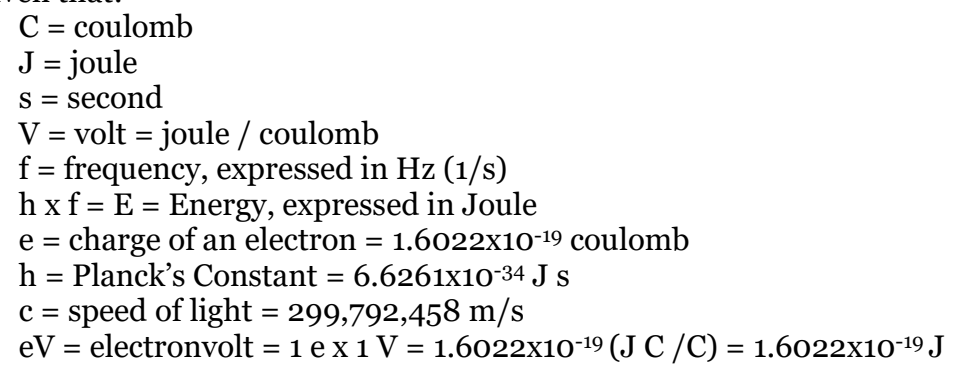

Therefore:

When $\mathrm{f}=1 \mathrm{~Hz}$, and from $\mathrm{E}=\mathrm{h} \mathrm{x}$, it follows that:

$6.6261 \times 10^{-34} \mathrm{~J}=$ energy of a photon with a frequency of $1 \mathrm{~Hz}$

Hence:

$1 \mathrm{eV}=6.6261 \times 10^{-34} \mathrm{~J} / 1.6022 \times 10^{-19} \mathrm{~J}=4.136 \times 10^{-15}$ photons with a frequency of $1 \mathrm{~Hz}$, or

$1 \mathrm{eV}=1$ photon of frequency $1 / 4.136 \times 10^{-15} \mathrm{~Hz}=0.242 \times 10^{-15} \mathrm{~Hz}=242 \mathrm{THz}$ (terahertz).

Furthermore, wavelength in a vacuum is:

$\lambda=\mathrm{c} / \mathrm{f}$

and so $242 \mathrm{THz}$ correspond to (in a vacuum) a wavelength equal to:

$\lambda=299,792,458 \mathrm{~m} / \mathrm{s} / 0.242 \times 10^{15} 1 / \mathrm{s}=1.239 \times 10^{-6} \mathrm{~m}=1.24 \mu \mathrm{m}$

Consequently. the wavelength in a vacuum of a photon with energy $1 \mathrm{eV}(\mathrm{f}=242 \mathrm{THz})$ is:

$1 \mathrm{eV} \rightarrow 1.24 \times 10^{-6} \mathrm{~m}=1.24 \mu \mathrm{m}$, which is a near-infrared wavelength.

In summary, energy - expressed in $\mathrm{eV}$ - of a photon of wavelength $\lambda$ equals $1.238 \times 10^{6} / \lambda$.

Example $1(\lambda=400 \mathrm{~nm}): 1.238 \times 10^{-6} / 0.4 \times 10^{-6}=3.1 \mathrm{eV}$

Example $2(\lambda=200 \mathrm{~nm}): 1.238 \times 10^{-6} / 0.2 \times 10^{-6}=6.2 \mathrm{eV}$

and, because it follows that $\lambda$ of a photon $=1.238 \times 10^{-6} /$ photon energy in $\mathrm{eV}$ :

Example $3(\mathrm{E}=1.24 \mathrm{eV}): 1.238 \times 10^{-6} / 1.14 \times 10^{-6}=1.1 \mu \mathrm{m}$ 